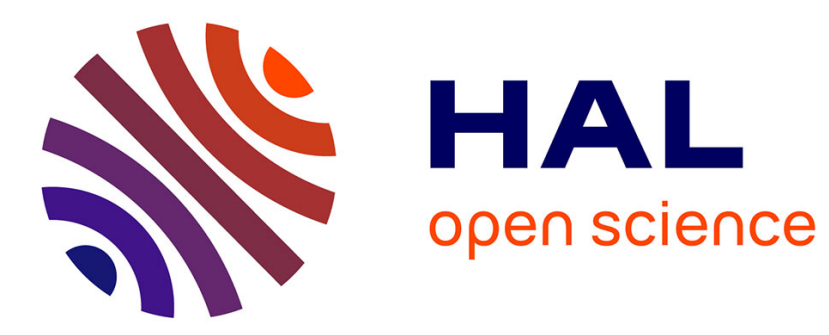

\title{
Reoptimization of the Maximum Weighted Pk-Free Subgraph Problem under Vertex Insertion
}

\author{
Nicolas Boria, Jérôme Monnot, Vangelis Paschos
}

\section{To cite this version:}

Nicolas Boria, Jérôme Monnot, Vangelis Paschos. Reoptimization of the Maximum Weighted Pk-Free Subgraph Problem under Vertex Insertion. 6th International Workshop on Algorithm and Computation (WALCOM 2012), Feb 2012, Dhaka, Bangladesh. pp.76-87, 10.1007/978-3-642-28076-4_10 . hal-01508825

\section{HAL Id: hal-01508825 \\ https://hal.science/hal-01508825}

Submitted on 14 Apr 2017

HAL is a multi-disciplinary open access archive for the deposit and dissemination of scientific research documents, whether they are published or not. The documents may come from teaching and research institutions in France or abroad, or from public or private research centers.
L'archive ouverte pluridisciplinaire HAL, est destinée au dépôt et à la diffusion de documents scientifiques de niveau recherche, publiés ou non, émanant des établissements d'enseignement et de recherche français ou étrangers, des laboratoires publics ou privés. 


\title{
Reoptimization of the MAX WEIGHTED $\boldsymbol{P}_{\boldsymbol{k}}$-FREE SUBGRAPH problem under vertex insertion*
}

\author{
Nicolas Boria ${ }^{1}$, Jérôme Monnot ${ }^{1}$, and Vangelis Th. Paschos ${ }^{1,2}$ \\ 1 LAMSADE, CNRS UMR 7243 and Université Paris-Dauphine \\ \{boria, monnot, paschos\}@lamsade.dauphine.fr \\ ${ }^{2}$ Institut Universitaire de France
}

\begin{abstract}
The reoptimization issue studied in this paper can be described as follows: given an instance $I$ of some problem $\Pi$, an optimal solution OPT for $\Pi$ in $I$ and an instance $I^{\prime}$ resulting from a local perturbation of $I$ that consists of insertions or removals of a small number of data, we wish to use OPT in order to solve $\Pi$ in $I^{\prime}$, either optimally or by guaranteeing an approximation ratio better than that guaranteed by an ex nihilo computation and with running time better than that needed for such a computation. In this setting we study the weighted version of MAX WEIGHTED $P_{k}$-FREE SUBGRAPH. We then show, how the technique we use allows us to handle also BIN PACKING.
\end{abstract}

\section{Introduction}

Hereditary problems in graphs, also known as maximal subgraph problems, include a wide range of classical combinatorial optimization problems, such as MAX WEIGHTED IndEPENDENT SET or MAX Weighted $H$-FreE SUBGRAPH. Most of these problems are known to be NP-hard, and even inapproximable within any constant approximation ratio unless $\mathbf{P}=\mathbf{N P}[16,17]$.

In what follows, we study approximation of such a problem, namely the maximum weight $P_{k}$-FREE SUbGRAPH, denoted by MAX WEIGHTED $P_{k}$-FREE SUBGRAPH, in the reoptimization setting, which can be described as follows: considering an instance $I$ of a given problem $\Pi$ with a known optimum OPT, and an instance $I^{\prime}$ which results from a local perturbation of $I$, can the information provided by OPT be used to solve $I^{\prime}$ in a more efficient way (i.e., with a lower complexity and/or with a better approximation ratio) than if this information wasn't available?

The reoptimization setting was introduced in [1] for METRIC TSP. Since then, many other optimization problems were discussed in this setting, including Steiner tree $[5,9,10,15]$, minimum Spanning tree [14], as well as various versions of TSP $[4,8,12]$. In all cases, the goal is to propose reoptimization algorithm that outperform their deterministic counterparts in terms of complexity

\footnotetext{
${ }^{*}$ Research supported by the French Agency for Research under the DEFIS program TODO, ANR-09-EMER-010
} 
and/or approximation ratio. In [7], the MAX WEIGHTED INDEPENDENT SET problem, as well as MIN WEIGHTED VERTEX COVER and MIN WEIGHTED SET COVER problems, are discussed in a similar setting up to the fact that perturbations there concerned the edge-set of the initial graph. The authors of [7] manage to provide optimal approximation results (i.e., upper and lower bounds matching ones the others) under the basic assumption that the initial solution is not necessarily optimal but $\rho$-approximate. Finally, let us note that in [6] reoptimization variants of the shortest common superstring problem are considered, where the local modifications consist of adding or removing a single string.

When one deals with hereditary problems, and $I^{\prime}$ results from a perturbation of the vertex set (insertion or deletion), solutions of $I$ remain feasible in $I^{\prime}$. This property is very interesting when reoptimizing hereditary problems, and makes most of them APX in the reoptimization setting. For exemple, a very simple algorithm provides a (1/2)-approximation for a whole class of hereditary problems, including MAX WEIGHTED INDEPENDENT SET when a single vertex is inserted [3]. Let us note that the unweighted versions of all these problems in this setting admit polynomial time approximation schemata. In what follows, we improve on this result by presenting algorithms designed for two specific hereditary problems, and also provide inapproximability bounds.

The paper is organized as follows: general properties regarding reoptimization and the MAX WEIGHTED $P_{k}$-FREE SUBGRAPH are presented in what follows in this section, while in Section 2 we present approximation and inapproximability results under vertex insertion for MAX WEIGHTED $P_{k}$-FREE SUBGRAPH. Our results are optimal (in the sense given two paragraphs above). To the best of our knowledge no such results exist in the reoptimization literature for the vertex insertion setting. In Section 3, the BIN PACKING problem is handled.

This paper is part of a larger work [13] devoted to the study of five maximum weight induced hereditary subgraph problems, namely, MAX WEIGHTED INDEPENDENT SET, MAX WEIGHTED $k$-COLORABLE SUBGRAPH, MAX WEIGHTED $P_{k}$-FREE SUBGRAPH, MAX WEIGHTED SPLIT SUBGRAPH and MAX PLANAR SUBGRAPH. For reasons of length limits some of the results are given without detailed proofs, the interested reader is referred to [13] where all proofs can be found.

Before presenting properties and results regarding reoptimization problems, we will first give formal definitions of what are reoptimization problems, reoptimization instances, and approximate reoptimization algorithms:

Definition 1. An optimization problem $\Pi$ is given by a quadruple $\left(\mathcal{I}_{\Pi}, S_{0} l_{\Pi}, m_{\Pi}\right.$, goal $\left.(\Pi)\right)$ where: $\mathcal{I}_{\Pi}$ is the set of instances of $\Pi$; given $I \in \mathcal{I}_{\Pi}$, $\operatorname{Sol}_{\Pi}(I)$ is the set of feasible solutions of $I$; given $I \in \mathcal{I}_{\Pi}$, and $S \in \operatorname{Sol}_{\Pi}(I)$, $m_{\Pi}(I, S)$ denotes the value of the solution $S$ of the instance $I ; \operatorname{goal}(\Pi) \in$ $\{\min , \max \}$.

A reoptimization problem $R \Pi$ is given by a pair $\left(\Pi, R_{R \Pi}\right)$ where: $\Pi$ is an optimization problem as defined in Definition $1 ; R_{R \Pi}$ is a rule of modification on instances of $\Pi$, such as addition, deletion or alteration of a given amount of data; given $I \in \mathcal{I}_{\Pi}$ and $R_{R \Pi}$, modif RI $\left(I, R_{R \Pi}\right)$ denotes the set of instances resulting from applying modification $R_{R \Pi}$ to $I$; notice that $\operatorname{modif}_{R \Pi}\left(I, R_{R \Pi}\right) \subset \mathcal{I}_{\Pi}$. 
For a given reoptimization problem $R \Pi\left(\Pi, R_{R \Pi}\right)$, a reoptimization instance $I_{R \Pi}$ of $R \Pi$ is given by a triple $\left(I, S, I^{\prime}\right)$, where: $I$ denotes an instance of $\Pi$, referred to as the initial instance; $S$ denotes a feasible solution for $\Pi$ on the initial instance $I ; I^{\prime}$ denotes an instance of $\Pi$ in $\operatorname{modif}_{R \Pi}\left(I, R_{R \Pi}\right) ; I^{\prime}$ is referred to as the perturbed instance. For a given instance $I_{R \Pi}\left(I, S, I^{\prime}\right)$ of $R \Pi$, the set of feasible solutions is $\operatorname{Sol}_{\Pi}\left(I^{\prime}\right)$.

Definition 2. For a given reoptimization problem $R \Pi\left(\Pi, R_{R \Pi}\right)$, a reoptimization algorithm $A$ is said to be a $\rho$-approximation reoptimization algorithm for $R \Pi$ if and only if: (i) A returns a feasible solution on all instances $I_{R \Pi}\left(I, S, I^{\prime}\right)$; (ii) A returns a $\rho$-approximate solution on all reoptimization instances $I_{R \Pi}\left(I, S, I^{\prime}\right)$ where $S$ is an optimal solution for $I$.

Note that Definition 2 is the most classical definition found in the literature, as well as the one used in this paper. However, an alternate (and more general) definition exists (used for example in $[5,7,9,10]$ ), where a $\rho_{1}$-approximation reoptimization algorithm for $R \Pi$ is supposed to ensure a $\rho_{1} \rho_{2}$-approximation on any reoptimization instance $I_{R \Pi}\left(I, S, I^{\prime}\right)$ where $S$ is a $\rho_{2}$-approximate solution in the initial instance $I$.

A property $\mathcal{P}$ on a graph is hereditary if the following holds: if the graph satisfies $\mathcal{P}$, then $\mathcal{P}$ is also satisfied by all its induced subgraphs. Following this definition, independence, planarity, bipartiteness are three examples of hereditary properties: in a given graph, any subset of an independent set is an independent set itself, and the same holds for planar and bipartite subgraphs. On the opposite hand, connectivity is no hereditary property since there might exist some subsets of $G$ whose removal disconnect the graph.

We denote by hereditary problem any problem that consists of finding the maximum set of vertices (in terms of cardinality or weight) that induces a subgraph verifying a given hereditary property.

A graph is said to be $P_{k}$-free if it does not contain a path on $k$ edges. Here, $P_{k}$-free means that the graph does not admit a $P_{k}$ as minor (and not as induced subgraph). A graph $H$ is a minor of a graph $G$, if $H$ can be obtained by a sequence of edge contractions and vertex or edge deletions. For example, a $C_{k+1}$ admits a $P_{k}$ as minor, so, although it does not admit a $P_{k}$ as induced subgraph, we consider that a $C_{k+1}$ is not $P_{k}$-free.

Let $G(V, E, w)$ be a vertex-weighted graph with $w(v) \geqslant 0$, for any $v \in V$. The MAX WEIGHTED $P_{k}$-FREE SUBGRAPH problem is the problem consisting, given a graph $G(V, E, w)$, of finding a subset $S$ of vertices such that $G[S]$ is $P_{k}$-free and maximizes $w(S)=\sum_{v \in S} w(v)$. For instance, MAX WeIGHTED INDEPENDENT SET is exactly the MAX WEIGHTED $P_{2}$-FREE SUBGRAPH problem, while MAX WEIGHTED $P_{3}$-FREE SUBGRAPH consists of finding an independent set and an induced matching of maximum total weight.

As it is proved in [16] (see Theorem 1 just below) most hereditary problems (hence MAX WEIGHTED $P_{k}$-FREE SUBGRAPH also) are highly inapproximable unless $\mathbf{P}=\mathbf{N P}$. 
Theorem 1. ([16]) There exists an $\varepsilon \in(0,1)$ such that MAX Weighted $P_{k^{-}}$ FREE SUBGRAPH cannot be approximated with ratio $n^{-\varepsilon}$ in polynomial time unless $\mathbf{P}=\mathbf{N P}$.

In the sequel, $G_{p}$ and $G_{p}^{\prime}$ will denote initial and perturbed instances, while $\mathrm{OPT}_{p}$ and $\mathrm{OPT}_{p}^{\prime}$ will denote optimal solutions in $G_{p}$ and $G_{p}^{\prime}$, respectively. For simplicity and when no confusion arises, we will omit subscript $p$. The function $w$ refers to the weight function, taking a vertex, a vertex set, or a graph as input (the weight of a graph is defined as the sum of weights of its vertices). Finally, note that throughout the whole paper, the term "subgraph" will always implicitly refer to "induced subgraph".

Under vertex insertion, the inapproximability bounds of Theorem 1 is easily broken. In [3], a very simple strategy, denoted by R1 in what follows, provides a (1/2)-approximation for any hereditary problem. This strategy consists of outputting the best solution among the newly inserted vertex and the initial optimum. Moreover, this strategy can also be applied when a constant number $h$ of vertices is inserted: it suffices to output the best solution between an optimum in the $h$ newly inserted vertices (that can be found in $O\left(2^{h}\right)$ through exhaustive search) and the initial optimum. The $1 / 2$ approximation ratio is also ensured in this case [3].

Note that an algorithm similar to R1 was proposed for KNAPSACK in [2]. Indeed, this problem, although not being a graph problem, it is hereditary in the sense defined above, so that returning the best solution between a newly inserted item and the initial optimum ensures a (1/2)-approximation ratio. The authors also show that any reoptimization algorithm that does not consider objects discarded by the initial optimal solution cannot have ratio better than $1 / 2$.

\section{MAX WEIGHTED $\boldsymbol{P}_{\boldsymbol{k}}$-FREE SUBGRAPH}

The max Weighted $P_{k}$-FreE SUbGraph problem discussed in this subsection refers to MAX WEIGHTED $P_{k}$-FREE SUBGRAPH (with $P_{k}$ as forbidden minor), and not to MAX WEIGHTED INDUCED $P_{k}$-FREE SUBGRAPH (with $P_{k}$ as forbidden induced subgraph). Formally, given a graph $G(V, E, w)$ and a constant $k \leqslant n$, the MAX WEIGHTED $P_{k}$-FREE SUBGRAPH problem handled in this section consists of finding a maximum-total weight set of vertices that induces a subgraph of $G$ that is $P_{k}$-free. The approximability analysis of MAX WEIGHTED $P_{k}$-FREE SUBGRAPH uses the following lemma whose proof can be found in [13].

Lemma 1. A $P_{k}$-free graph can be colored with $k$ colors in polynomial time.

Proposition 1. Under one vertex insertion, MAX WeIGHTED $P_{k}$-FREe SUBGRAPH is inapproximable within ratio $\frac{2 k}{3 k+1}+\varepsilon$ in polynomial time if $k$ is odd, and inapproximable within ratio $\frac{2 k}{3 k+2}+\varepsilon$ if $k$ is even, unless $\mathbf{P}=\mathbf{N P}$.

Proof (Sketch). The technique used for the proof can be sketched as follows. Considering an unweighted graph $H(V, E)$ on which one wants to solve a given 
hereditary problem $\Pi$, known to be inapproximable within any constant ratio, we build a reoptimization instance $I_{p}$, where $p$ denotes a vector of fixed size (i.e., independent of the size $n$ of $G$; so, $|p|$ is a fixed constant) that contains integer parameters between 1 and $n$. This instance is characterized by an initial graph $G_{p}$ (that contains $H$ ), with a known solution, and a perturbed instance $G_{p}^{\prime}$.

Then, we prove that, for some specific (yet unknown) value $p^{\prime}$ of the parameter vector $p$ (that is, when the value of $p$ coincides with some unknown structural parameters of the graph, like independence number for example), an optimal solution can be easily determined in the initial graph $G_{p^{\prime}}$, and a $\rho$-approximate solution $S_{p^{\prime}}$ in $G_{p^{\prime}}^{\prime}$ necessarily induces a solution $S_{p^{\prime}}[V]$ in $H$, that is a constant approximation for the initial problem. Considering that the vector $p$ can take at most $n^{|p|}$ possible values, it is possible in polynomial time to build all instances $I_{p}$, to run the polynomial $\rho$-approximation algorithm on all of them, and to return the best set $S_{p^{*}}[V]$ as solution for $\Pi$ in $H$. The whole procedure is polynomial and ensures a constant-approximation for $\Pi$, which is impossible unless $\mathbf{P}=\mathbf{N P}$, so that a $\rho$-approximation algorithm cannot exist for the considered reoptimization version of $\Pi$, unless $\mathbf{P}=\mathbf{N P}$.

Consider now a connected graph $H$ that has independence number $\alpha$, on which one wishes to solve (or approximate) MAX WEIGHTED INDEPENDENT SET problem. Transform $H$ into a graph $H_{k}(V, E)$ in the following way: each vertex $v_{i}$ of $H$ is turned into a clique $V_{i}$ of $k$ vertices in $H_{k}$; if $\left(v_{i}, v_{j}\right)$ belongs to $H$, then all edges between vertices of cliques $V_{i}$ and $V_{j}$ are in $E$. Note that $H_{k}$ is connected as $H$ itself is also connected. Considering that the biggest independent set in $H$ has size $\alpha$, then the same holds in $H_{k}$ : an independent set in $G$ can only take one vertex in each clique $V_{i}$, and it cannot take two vertices from cliques that corresponds to neighbors in $H$.

Following Lemma 1 , any $P_{k}$-free subgraph in $H_{k}$ can be partitioned in $k$ independent sets, so that, denoting by OPT an optimal $P_{k}$-free subgraph in $H_{k}$, $|\mathrm{OPT}| \leqslant k \alpha$. Moreover, denoting by $I S$ an optimal independent set in $H$ (that has exactly $\alpha$ vertices), then, in $H_{k}$, the union of all cliques corresponding to vertices of $I S$ induces a $P_{k}$-free subgraph with value exactly $k \alpha$, so that $|\mathrm{OPT}|=$ $k \alpha$.

Remark 1. Following the same arguments, it holds that for any $i \leqslant k$ a $P_{i}$-free subgraph in $H_{k}$ cannot have weight more than $i \alpha$ (and an optimal one has weight exactly $i \alpha)$.

First, suppose that $k$ is odd. We build a weighted reoptimization instance $I_{\alpha, k}$ of MAX Weighted $P_{k}$-FreE SUbGraph as follows (Figure 1 provides a representation of the general structure).

- The initial graph $G_{\alpha, k}$ is obtained by adding to the graph $H_{k}$ a set $X$ of vertices which consists of two cliques $X_{1}$, and $X_{2}$ to $G$. Both these cliques have $k$ vertices, each with weight $k \alpha$. Clique $X_{1}$, is divided into two subcliques $X_{1 C}$ and $X_{1 N C} ; X_{1 C}$ has $(k+1) / 2$ vertices that are all connected to all vertices in $V$, while the other $(k-1) / 2$ vertices of $X_{1 N C}$ are not connected to any vertex in $V$. Clique $X_{2}$ is divided in the same manner. Finally, each vertex in $V$ receives 


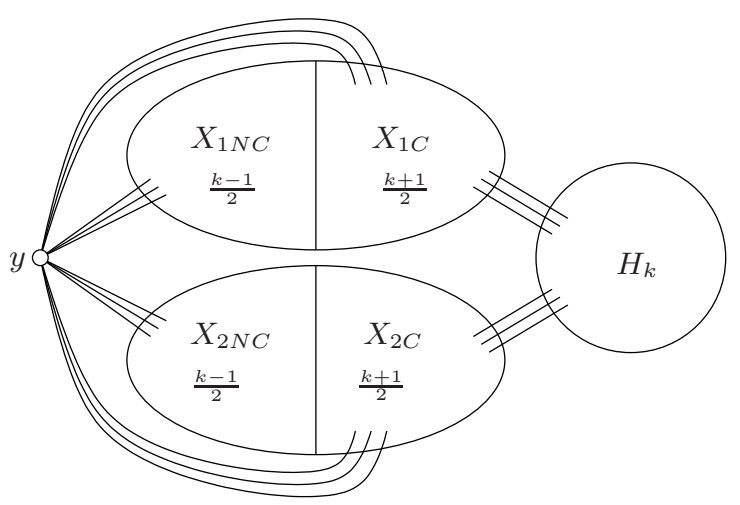

Fig. 1. Reoptimization instance $I_{\alpha, k}$

weight $k+1$, hence, for any $1 \leqslant i \leqslant k$ an optimal $P_{i}$-free subgraph inside $V$ has weight exactly $i(k+1) \alpha$.

- The perturbed graph $G_{\alpha, k}^{\prime}$ is obtained by adding a single vertex $y$ to $G_{\alpha}$, with weight $k(k+1) \alpha$, that is connected to all vertices of $X$.

We first prove that $X$ defines an optimum on the initial graph $G_{\alpha, k}$, so that the reoptimization instance $I_{\alpha, k}$ is well defined. Then, we show that $\{y\} \cup X_{1 N C} \cup$ $X_{2 N C} \cup F^{*}$ (where $F^{*}$ is an optimum in $H_{k}$ ) is feasible in the graph $G_{\alpha, k}^{\prime}$, so that $w\left(\mathrm{OPT}^{\prime}\right) \geqslant w\left(\{y\} \cup X_{1 N C} \cup X_{2 N C} \cup F^{*}\right)=k(3 k+1) \alpha$. This lower bound on the weight of the optimum, naturally induces a lower bound on the weight of a $\left(\frac{2 k}{3 k+1}+\varepsilon\right)$-approximate solution, say $S_{\alpha, k}: w\left(S_{\alpha, k}\right) \geqslant\left(\frac{2 k}{3 k+1}+\varepsilon\right) w\left(\mathrm{OPT}^{\prime}\right) \geqslant$ $(1+\varepsilon) 2 k^{2} \alpha$.

We then show that any feasible solution on the subgraph induced by $\{y\} \cup X$ cannot have weight more than $2 k^{2} \alpha$, and this holds a fortiori for the restriction of $S_{\alpha, k}$ to this subgraph, denoted by $S_{\alpha, k}[\{y\} \cup X]$. Combining this fact with the expression for $w\left(S_{\alpha, k}\right)$ above, and taking into account that $\{\{y\} \cup X, V\}$ defines a partition of the final graph, we derive that $w\left(S_{\alpha, k}[V]\right)=w\left(S_{\alpha, k}\right)-$ $w\left(S_{\alpha, k}[\{y\} \cup X]\right) \geqslant 2 \varepsilon k^{2} \alpha$. Hence, a $\left(\frac{2 k}{3 k+1}+\varepsilon\right)$-approximation algorithm for the reoptimization version of MAX WEIGHTED $P_{k}$-FREE SUBGRAPH can be used to derive a polynomial $\varepsilon$-approximation algorithm for the static version of MAX Weighted IndEPENDENT SET, which is impossible unless $\mathbf{P}=\mathbf{N P}$.

In the same way, we present a $\left(\frac{2 k}{3 k+2}+\varepsilon\right)$ inapproximability bound for the case where $k$ is even. The structure of the instance is somewhat more complex, but the structure of the proof itself is similar. A full proof of both inapproximability bounds can be found in [13].

Remark 2. The proof of Proposition 1 works also even if we assume that a $\rho$ approximate solution is given instead of an optimal one. In this case, the bounds claimed in Proposition 1 are simply multiplied by $\rho$. 
We now prove that ratio of Proposition 1 is tight for small values of $k$, namely when $k \leqslant 6$. Consider the following hypothesis.

Hypothesis 1. In polynomial time, a $P_{k}$-free subgraph $S$ can be partitioned in 3 sets $S_{1}, S_{2}$ and $S_{3}$, such that both $S_{1}$ and $S_{2}$ are $P_{\lceil k / 2\rceil-1}$-free, and $w\left(S_{3}\right) \leqslant$ $w(S) / k$ if $k$ is odd and $w\left(S_{3}\right) \leqslant 2 w(S) / k$ if $k$ is even.

Proposition 2. For values of $k$ for which Hypothesis 1 is true, MAX WEIGHTED $P_{k}$-FREE SUBGRAPH problem is approximable within ratio $\frac{2 k}{3 k+1}$ for odd values of $k$, and $\frac{2 k}{3 k+2}$ for even values of $k$ in the reoptimization setting, under one vertex insertion.

Proof. Consider a reoptimization instance $I$ of MAX Weighted $P_{k}$-FreE SUBGRAPH, given by two graphs $G$ (initial) and $G^{\prime}$ (perturbed) and an optimal solution OPT on the initial graph $G$. Graphs $G$ and $G^{\prime}$ differ only by vertex $y$ (and its incident edges) which belongs to $G^{\prime}$ but not to $G$. Classically, denoting by $\mathrm{OPT}^{\prime}$ an optimal solution on $G^{\prime}$, it holds that $w(\mathrm{OPT}) \geqslant w\left(\mathrm{OPT}^{\prime}\right)-w(y)$. Suppose that Hypothesis 1 is verified, and consider the following algorithm: partition OPT in 3 sets $S_{1}, S_{2}$ and $S_{3}$ as defined in Hypothesis 1, and without loss of generality, suppose $w\left(S_{1}\right) \geqslant w\left(S_{2}\right)$; set $\mathrm{SOL}_{1}=S_{1} \cup\{y\}$ and $\mathrm{SOL}_{2}=\mathrm{OPT}$; return the best solution $\mathrm{SOL}$ between $\mathrm{SOL}_{1}$ and $\mathrm{SOL}_{2}$.

First, let us prove that this algorithm returns a feasible solution: $\mathrm{SOL}_{2}$ is trivially feasible in $G^{\prime}$, and consider a path $P$ in $\mathrm{SOL}_{1}$. If this path does not go through $y$ then it cannot go through more than $\lceil k / 2\rceil-1$ vertices (by hypothesis, $S_{1}$ is $P_{\lceil k / 2\rceil-1}$-free). If it does go through $y$, then denote by $P_{1}$ the set of vertices visited before $y$ in $P$ and $P_{2}$ the set of vertices visited after. Considering that both $P_{1}$ and $P_{2}$ are included in $S_{1}$, which is supposed to be $P_{\lceil k / 2\rceil-1}$-free, then both $\left|P_{1}\right|,\left|P_{2}\right| \leqslant\lceil k / 2\rceil-1$, so that $|P|=\left|P_{1}\right|+\left|P_{2}\right|+1 \leqslant k$, and thus $\mathrm{SOL}_{1}$ is also $P_{k}$-free.

Let $r_{k}$ be an integer that is equal to 1 if $k$ is odd, and to 2 if $k$ is even. Regarding the partitioning induced by Hypothesis 1 , it holds that $w\left(S_{3}\right) \leqslant \frac{r_{k} w(\mathrm{OPT})}{k}$, and thus $w\left(S_{1}\right) \geqslant\left(w(\mathrm{OPT})-w\left(S_{3}\right)\right) / 2 \geqslant \frac{k-r_{k}}{2 k} w(\mathrm{OPT})$, thus $w\left(\mathrm{SOL}_{1}\right) \geqslant$ $\frac{k-r_{k}}{2 k} w(\mathrm{OPT})+w(y)=\frac{k-r_{k}}{2 k} w\left(\mathrm{OPT}^{\prime}\right)+\frac{k+r_{k}}{2 k} w(y)$.

On the other hand, $w\left(\mathrm{SOL}_{2}\right) \geqslant w\left(\mathrm{OPT}^{\prime}\right)-w(y)$. Summing expressions for $w\left(\mathrm{SOL}_{1}\right)$ and $w\left(\mathrm{SOL}_{2}\right)$ with coefficients 1 and $\frac{k+r_{k}}{2 k}$ respectively, one finally proves that $\frac{3 k+r_{k}}{2 k} w(\mathrm{SOL}) \geqslant w\left(\mathrm{SOL}_{2}\right)+\frac{k+r_{k}}{2 k} w\left(\mathrm{SOL}_{1}\right) \geqslant w\left(\mathrm{OPT}^{\prime}\right)$.

Note once more that, as it can be seen from the proof of Proposition 2, Remark 2 always holds.

Proposition 3. Hypothesis 1 holds for $k \leqslant 6$.

Proof (Sketch). In what follows, we suppose that the $P_{k}$-free graph $S$ to be partitioned is connected (if it is not so, then proving that the hypothesis is true for each of its connected components amounts to proving it for the whole graph).

$\mathbf{k}=\mathbf{1}, \mathbf{2}$. Simply set $S_{3}=S$ and $S_{1}, S_{2}=\emptyset$. 
$\mathbf{k}=3$. Split the graph in three independent sets $S_{1}^{\prime}, S_{2}^{\prime}$, and $S_{3}^{\prime}$ in polynomial time (that is possible according to Lemma 1), and w.l.o.g., suppose $w\left(S_{1}^{\prime}\right) \geqslant$ $w\left(S_{2}^{\prime}\right) \geqslant w\left(S_{3}^{\prime}\right)$. Finally, set $S_{1}=S_{1}^{\prime}, S_{2}=S_{2}^{\prime}$, and $S_{3}=S_{3}^{\prime}$.

$\mathbf{k}=4$. Split the graph in four independent sets from $S_{1}^{\prime}$ to $S_{4}^{\prime}$ in polynomial time, and w.l.o.g., suppose $w\left(S_{1}^{\prime}\right) \geqslant w\left(S_{2}^{\prime}\right) \geqslant w\left(S_{3}^{\prime}\right) \geqslant w\left(S_{4}^{\prime}\right)$. Finally, set $S_{1}=$ $S_{1}^{\prime}, S_{2}=S_{2}^{\prime}$, and $S_{3}=S_{3}^{\prime} \cup S_{4}^{\prime}$.

$\mathbf{k}=\mathbf{5}$. This case requires a rather long and involved analysis, which could not be included in the paper due to length limits. A full proof can be found in [13].

$\mathbf{k}=6$. In this case, Hypothesis 1 is verified if $S$ can be partitioned in $3 P_{2}$ free subgraphs (the lightest of which will be $S_{3}$, and the other two $S_{1}$ and $S_{2}$ ). Here, we distinguish the following four cases.

Case 1. $S$ contains a $C_{6}$. It is clear that $S$ contains exactly 6 vertices, which can easily be partitioned in three $P_{2}$-free subgraphs ( 2 vertices in each set of the partition).

Case 2. $S$ contains a $C_{5}$ and no $C_{6}$. Let $S^{\prime}$ denote the set of vertices that are not in $C_{5}$. It holds that $S^{\prime}$ is an independent set, and that a pair of vertices that are neighbors in the $C_{5}$ cannot both have neighbors in $S^{\prime}$ (if one of these two properties is not verified, then there exists a $P_{6}$ in $S$. Thus, without making assumption on the number of chords in $C_{5}$, the general structure of $S$ is as described in Figure 2, with a partitioning in three $P_{2}$-free subgraphs (represented in the figure as white, grey, and black vertices).

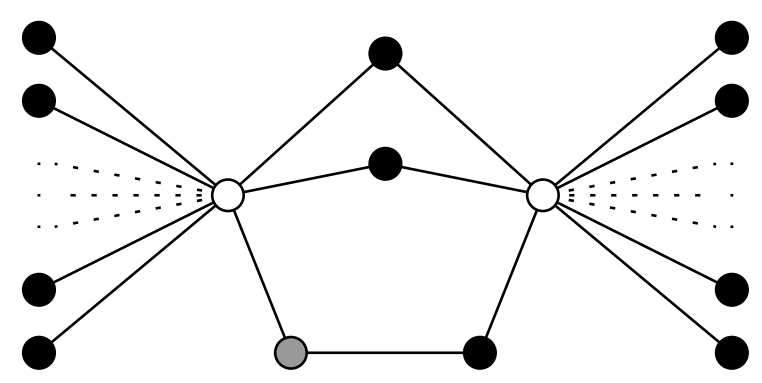

Fig. 2. Partition of $P_{6}$-free graph in $3 P_{2}$-free subgraphs in Case 2

Case 3. $S$ contains a $C_{4}$. We distinguish here the following two subcases.

Case 3-a. $S$ contains a $C_{4}$ but no $C_{5}$ and no $C_{6}$, and denoting by $S^{\prime}$ the set of vertices that are not in the $C_{4}, S^{\prime}$ contains at least an edge $\left(v_{1}, v_{2}\right)$. Obviously, these two vertices $v_{1}$ and $v_{2}$ cannot be connected to two different vertices of the $C_{4}$, otherwise, $S$ would contain a $C_{5}$ or a $C_{6}$.

Moreover, at least one of these two vertices must be connected to a vertex of the $C_{4}$. Indeed, taking into account that $S$ is supposed to be connected, if none of these vertices is connected to a vertex of the $C_{4}$, then there exists a $P_{6}$ in $S$. 
Finally, supposing w.l.o.g that $v_{1}$ is the vertex connected to a vertex $x$ in the $C_{4}$, then no neighbor of $x$ in the $C_{4}$ can be connected to any vertex of $S^{\prime}$ (otherwise there would exist a $P_{6}$ in $S$ ).

Hence, denoting vertices of the $C_{4}$ by $x, y, z$ and $t$ as in Figure 3, only vertices $x$ and $z$ might have neighbors in $S^{\prime}$. Moreover, denoting by $N(\{x\} \cup\{z\})$ the neighborhood of these two vertices, it holds that this set is $P_{2}$ free, otherwise a path on 7 vertices would exist in $S$ (the 3 vertices of the $P_{2}$ in $N(\{x\} \cup\{z\}$ ), and the 4 vertices of the $\left.C_{4}\right)$. Thus, coloring in white the vertices of $N(\{x\} \cup\{z\})$ as well as $y$ and $t$, and in black all the other vertices of $S$, one gets a partition of $S$ into two $P_{2}$ free colors.

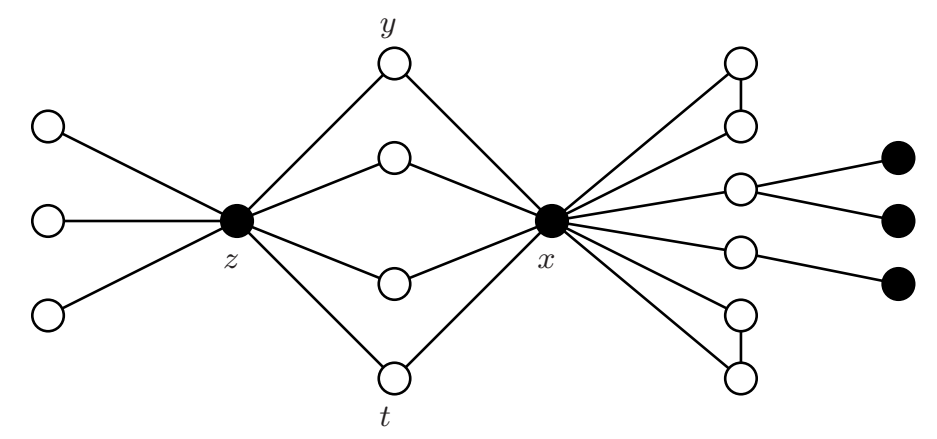

Fig. 3. Partition of $P_{6}$-free graph in $2 P_{2}$-free subgraphs in Case 3 -a

Case 3-b. $S$ contains a $C_{4}$ but no $C_{5}$ and no $C_{6}$, and let $S^{\prime}$ denote the set of vertices that are not in the $C_{4}, S^{\prime}$ contains no edge. This case is much simpler than the previous one, considering that $S^{\prime}$ forms an independent set. To get a partition of $S$ into three $P_{2}$-free subgraphs, it suffices to consider $S^{\prime}$ itself as the first subset of the partition, $\{x\} \cup\{z\}$ as the second, and $\{y\} \cup\{t\}$ as the third.

Case 4. $S$ contains no $C_{4}$, no $C_{5}$ and no $C_{6}$. In this case, it holds that the neighborhood $N(x)$ of any vertex $x$ is $P_{2}$-free, since a $P_{2}$ (on 2 edges, and 3 vertices) in $N(x)$ amounts to a $C_{4}$ in $x \cup N(x)$. Moreover, denoting by $\left.N(N(x)))=N^{2}(x)\right)$ the set of neighbors of vertices in $N(x)$, it holds that $N(N(x))$ is also $P_{2}$-free, since the neighborhood of each vertex in $N(x)$ is $P_{2}$-free (considering what we stated above), and disjoint from one another (otherwise there would exist a $C_{4}$ in $S$ ). Naturally, the same holds for any $N^{i}(x)$.

Hence, in this case, the graph can be easily partitioned in $2 P_{2}$-free subgraphs: starting from a arbitrary vertex that is colored black, one colors its neighborhood white, then the neighborhood of its neighborhood black, etc. Considering what we proved earlier, each color defines a $P_{2}$-free subgraph.

Finally, Hypothesis 1 is also verified when $k=6$. 


\section{Reoptimization and BIN PACKING}

Given a constant $B$, a list $L$ of $n$ items $L=(1,2, \ldots, n)$, such that, for any $i=1, \ldots, n$, its size $a_{i} \leqslant B$, and $n$ bins each of capacity $B$, the BIN PACKING problem consists of arranging the items of $L$ in the bins without exceeding their capacity (i.e., the sum of the sizes of the items placed in every bin must not exceed $B$ ) and in such a way that a minimum number of bins is used.

We show in this section that the basic technique used before in order to get inapproximability results can be also applied on hereditary problems not necessarily defined on graphs. This is, for instance the case of BIN PACKING. We will prove that this problem is inapproximable within approximation ratio $3 / 2-\varepsilon$, for any $\varepsilon>0$, in the reoptimization setting studied in this paper. For simplicity we consider a non-normalized instance of BIN PACKING where item $i$, $i \leqslant n$ has integer size $a_{i}$ and bins have capacity $B$. We also assume that, for every $i, a_{i}<B$.

Suppose, ad contrario, that BIN PACKING is approximable within approximation ratio $3 / 2$ under items insertion and consider an instance $I$ of the PARTITION problem, where $n+1$ items $0,1, \ldots, n$ with sizes $a_{0}, a_{1}, \ldots, a_{n}$ are given such that, for $i=0,1, \ldots, n, a_{i}<B$ and $\sum_{i=0}^{n} a_{i}=2 B$, and the objective is to find a partition of the items (if any) into two subsets such that the sum of the sizes of their items is equal to $B$. PARTITION is known to be NP-complete.

Assume now that items are ordered in decreasing size order (i.e., $a_{0} \geqslant a_{1} \geqslant$ $\left.\ldots \geqslant a_{n}\right)$ and consider the list of items $L=\left(a_{1}, \ldots, a_{n}\right)$ as instance of BIN PACKING. Obviously, since $a_{0}<B$ and $\sum_{i=0}^{n} a_{i}=2 B$, it holds that $\sum_{i=1}^{n} a_{i}>B$. Thus, an optimal BIN PACKING-solution for $L$ has value greater than 1 . We claim that $L$ has a solution using 2 bins. Indeed, such a solution places the first $k$ items in one bin, where $k$ is the largest index such that $\sum_{i=1}^{k} a_{i} \leqslant B$, and the rest of the items from $k+1$ to $n$ in a second bin. Let us prove that such a solution is feasible, or equivalently, that $\sum_{i=k+1}^{n} \leqslant B$. Assume, ad contrario, that $\sum_{i=k+1}^{n} a_{i}>B$, recall that, by the definition of $k, \sum_{i=1}^{k+1} a_{i}>B$ and observe that $\sum_{i=0}^{k} a_{i} \geqslant \sum_{i=1}^{k+1} a_{i}>B$. In other words, on the hypothesis that $\sum_{i=k+1}^{n} a_{i}>B$, we derive that $\sum_{i=0}^{n} a_{i}=\sum_{i=0}^{k} a_{i}+\sum_{i=k+1}^{n} a_{i}>B+B=2 B$, a contradiction. So, the BIN PACKING-instance $L$ has a solution of 2 bins. In all, we can assume that the initial BIN PACKING-instance is $L$ and the optimal solution provided with is as just described.

Assume now that item 0 with size $a_{0} \geqslant a_{1}$ arrives. On the hypothesis of the existence of a polynomial reoptimization algorithm achieving approximation ratio $3 / 2-\varepsilon$ for BIN PACKING, if the instance $I$ of PARTITION is a "yes"-instance, then this algorithm would provide a solution with 2 bins, while if $I$ is a "no"instance, the algorithm would provide a solution with at least 3 bins, deciding so in polynomial time PARTITION.

On the other hand, BIN PACKING is immediately approximable within $3 / 2$ in the reoptimization setting under consideration. Given an instance $L$ and a solution with $k$ bins, when an element arrives, one can open a new bin in order to place it (after, eventually, a quick check that all the items cannot be placed in 
the same bin) guaranteeing so an approximation ratio $(k+1) / k \leqslant 3 / 2$, for $k \geqslant 2$, while the case $k=1$ is trivially polynomial (just check whether $\sum_{i=1}^{n} a_{i} \leqslant B$, or not).

\section{Conclusion}

We have discussed the approximability of MAX WEIGHTED $P_{k}$-FREE SUBGRAPH in the reoptimization setting under vertex insertion. It appears that the initial optimum (or a good initial solution) provides a very useful information when approximating the modified instances, and we presented reoptimization algorithms which take advantage of this information in the best possible way, since the approximation ratio provided is the best constant ratio achievable in polynomial time (unless $\mathbf{P}=\mathbf{N P}$ ). In this paper, we have proved optimal results for MAX WEIGHTED $P_{k}$-FREE SUBGRAPH even if there exist 2 different weights 1 and $\mathrm{M}$ in the instance. We can easily prove that MAX WEIGHTED $P_{k}$-FREE SUBGRAPH has a PTAS for any fixed $k$. Moreover, we can adapt the proofs given in the paper to produce results depending on parameter $M$ as done in [7]. Finally, in [11], the authors prove that the knowledge of all optimal solutions for free doesn't help TSP reoptimization. It is interesting to handle this question for MAX WEIGHTED $P_{k}$-FREE SUBGRAPH.

\section{References}

1. C. Archetti, L. Bertazzi, and M.G. Speranza. Reoptimizing the traveling salesman problem. Networks, 42(3):154-159, 2003.

2. C. Archetti, L. Bertazzi, and M.G. Speranza. Reoptimizing the 0-1 knapsack problem. Discrete Applied Mathematics, 158(17):1879-1887, 2010.

3. G. Ausiello, V. Bonifaci, and B. Escoffier. Complexity and approximation in reoptimization. CiE 200\%: Logic and Computation and Logic in the Real World, 2007.

4. G. Ausiello, B. Escoffier, J. Monnot, and V.Th. Paschos. Reoptimization of minimum and maximum traveling salesman's tours. In Lars Arge and Rusins Freivalds, editors, SWAT, volume 4059 of Lecture Notes in Computer Science, pages 196-207. Springer, 2006.

5. D. Bilò, H.-J. Böckenhauer, J. Hromkovic, R. Královic, T. Mömke, P. Widmayer, and A. Zych. Reoptimization of steiner trees. In Joachim Gudmundsson, editor, SWAT, volume 5124 of Lecture Notes in Computer Science, pages 258-269. Springer, 2008.

6. D. Bilò, H.-J. Böckenhauer, D. Komm, R. Královi, T. Mömke, S. Seibert, and A. Zych. Reoptimization of the shortest common superstring problem. In G. Kucherov and E. Ukkonen, editors, CPM'09, volume 5577 of Lecture Notes in Computer Science, pages 78-91. Springer-Verlag, 2009.

7. D. Bilò, P. Widmayer, and A. Zych. Reoptimization of weighted graph and covering problems. In Evripidis Bampis and Martin Skutella, editors, WAOA, volume 5426 of Lecture Notes in Computer Science, pages 201-213. Springer, 2008. 
8. H.-J. Böckenhauer, L. Forlizzi, J. Hromkovic, J. Kneis, J. Kupke, G. Proietti, and P. Widmayer. On the approximability of tsp on local modifications of optimally solved instances. Algorithmic Operations Research, 2(2):83-93, 2007.

9. H.-J. Böckenhauer, J. Hromkovic, R. Královic, T. Mömke, and P. Rossmanith. Reoptimization of steiner trees: Changing the terminal set. Theor. Comput. Sci., 410(36):3428-3435, 2009.

10. H.-J. Böckenhauer, J. Hromkovic, T. Mömke, and P. Widmayer. On the hardness of reoptimization. In Viliam Geffert, Juhani Karhumäki, Alberto Bertoni, Bart Preneel, Pavol Návrat, and Mária Bieliková, editors, SOFSEM, volume 4910 of Lecture Notes in Computer Science, pages 50-65. Springer, 2008.

11. H.-J. Böckenhauer, J. Hromkovič, and A. Sprock. Knowing all optimal solutions does not help for TSP reoptimization. In J. Kelemen and A. Kelemenova, editors, Computation, Cooperation, and Life: Essays Dedicated to Gheorghe Paun on the Occasion of His 60th Birthday, volume 6610 of Lecture Notes in Computer Science, pages 7-15. Springer-Verlag, 2011.

12. H.-J. Böckenhauer and D. Komm. Reoptimization of the metric deadline TSP. J. Discrete Algorithms, 8(1):87-100, 2010.

13. N. Boria, J. Monnot, and V. Th. Paschos. Reoptimization of maximum weight induced hereditary subgraph problems. Cahier du LAMSADE 311, LAMSADE, Universit Paris-Dauphine, Juin 2001.

14. N. Boria and V.Th. Paschos. Fast reoptimization for the minimum spanning tree problem. Journal of Discrete Algorithms, 8(3):296-310, 2010.

15. B. Escoffier, M. Milanic, and V.Th. Paschos. Simple and fast reoptimizations for the steiner tree problem. Algorithmic Operations Research, 4(2):86-94, 2009.

16. C. Lund and M. Yannakakis. The approximation of maximum subgraph problems. Automata, Languages and Programming, pages 40-51, 1993.

17. D. Zuckerman. Linear degree extractors and the inapproximability of max clique and chromatic number. In STOC'06, pages 681-690, 2006. 